\title{
Ultrasound molecular imaging of atherosclerosis
}

\author{
Beat A. Kaufmann* \\ Division of Cardiology, University Hospital Basel, Petersgraben 4, 4031 Basel, Switzerland \\ Received 18 February 2009; revised 15 April 2009; accepted 27 April 2009; online publish-ahead-of-print 3 June 2009
}

Time for primary review: 26 days

KEYWORDS

Atherosclerosis;

Ultrasound;

Contrast;

Molecular imaging
Recent advances in our understanding of the pathophysiological mechanisms of atherosclerosis have created the need for better non-invasive imaging of vascular phenotype. Ultrasound is widely available, inexpensive, and well suited for high-throughput screening in populations that are at risk for atherosclerosis. Novel ultrasonic approaches for the diagnosis of vascular changes in atherosclerosis include (1) assessment of plaque composition by evaluation of the backscattering properties of tissue, (2) assessment of the changes in arterial wall biomechanics, (3) assessment of plaque neovascularization, and (4) molecular imaging of vascular phenotype changes on a subcellular level. It is thought that such new imaging methodologies will lead to earlier detection of atherosclerosis, and better assessment of the risk for aggressive disease progression. Novel therapies for atherosclerosis will undoubtedly become available within the next decades, and non-invasive imaging techniques will be needed for cost-efficient application of existing and new drugs.

\section{Introduction}

Despite advances in primary and secondary prevention, ${ }^{1}$ cardiovascular diseases remain the number one killer in western countries. ${ }^{2}$ In addition, the decreases in mortality rates have been levelling off in recent years in young adults, ${ }^{1}$ while the prevalence of obesity and the metabolic syndrome are increasing. ${ }^{3,4}$ Thus, it is estimated that currently up to $40 \%$ of the adult population in the USA would fall into an intermediate risk category with a 6-20\% risk of developing symptomatic coronary heart disease within the ensuing 10 years. ${ }^{5}$ Therefore, methods for further risk stratification of individuals at intermediate risk are needed to make more efficient use of the existing and future prevention therapies.

Non-invasive imaging has developed rapidly in the last years, and for all major imaging techniques (computed tomography, single photon emission computed tomography, positron emission tomography, magnetic resonance, ultrasound) recent advances have been used to better evaluate cardiovascular disease. For the assessment of atherosclerotic disease, these methods have been used to look at plaque morphology and composition, biomechanical properties of vessels, or the consequences of plaque development on blood flow or blood flow reserve. The ultimate goals of all these efforts are three-fold: (1) earlier detection of pathophysiological changes known to be involved in the development of atherosclerosis; (2) better estimation of the risk of rapid disease progression; and (3) gauge the risk for

* Corresponding author. Tel: +41 6132867 12; fax: +4161 2654598 .

E-mail address: kaufmannb@uhbs.ch complications in established atherosclerosis. Given the fact that atherosclerosis is a complex, chronic disorder involving inflammatory and proliferative signalling pathways, it is generally thought that imaging of molecular events during all stages of the pathogenesis of atherosclerosis could contribute to better and earlier diagnostic assessment, risk estimation, and measurement of treatment effects. A basic requirement for non-invasive imaging tests in risk-stratification for cardiovascular disease is that they should provide incremental information over the assessment of traditional risk factors. Ideally, the same imaging techniques should also be able to gauge the influence of treatment strategies on disease progression and risk modification. Whether an individual imaging strategy will eventually be incorporated into risk-assessment algorithms will depend on the strength of the additional information provided relative to affordability, availability, and ease of use of the method. Ultrasound imaging is widely available and could be used for high-throughput screening at low cost, which compares favourably with other imaging techniques when being considered as a screening tool in large populations at risk for atherosclerotic events. This review will briefly discuss conventional ultrasound imaging methods for the assessment of atherosclerosis with an emphasis on newer emerging techniques, and then mainly focus on recent developments in ultrasound molecular imaging of atherosclerosis.

\section{Histomorphometric imaging}

Non-invasive ultrasound imaging of morphological changes related to atherosclerosis has to rely on assessing changes 
in vessels other than the coronary tree. The general assumption is that changes in vessels like the carotid artery that are easily imaged can serve as surrogate markers for both atherosclerotic changes in the coronaries and future risk of cardiovascular events. In its simplest form, B-mode ultrasound has been used to assess different measures of plaque burden such as the sum of the maximal thickness of protruding plaques visualized, or the sum of the total area of all plaques in the carotid arteries. Plaque burden measured in elderly men and in patients with known coronary artery disease has been shown to be an independent predictor of future cardiovascular mortality and coronary events. ${ }^{6,7}$ Quite obviously, the clinical utility of a simple assessment of plaque burden has limitations. First, only changes that occur relatively late during the disease process can be imaged, and thus the window of opportunity for preventive treatments is likely limited. Secondly, B-mode ultrasound has a limited ability to assess the biological characteristics of plaques that are known to influence the susceptibility to cardiovascular events. The first of these limitations has been addressed by measuring morphological changes in the vascular intima that occur during the relatively early stages of the pathogenesis of atherosclerosis. Changes in intimal thickness are most commonly measured as the carotid intimal-medial thickness (CIMT), as on B-mode ultrasound images only the luminal-intimal interface and the medial-adventitial interface can reliably be identified. Several large observational studies have shown that CIMT is associated with established risk factors for coronary heart disease and that it is an independent predictor of cardiovascular events. ${ }^{8-12}$ However, so far, there have been no studies published to show that measurements of CIMT provide incremental prognostic value over the assessment of traditional risk factors in individual patients. In the aforementioned observational studies, the differences in CIMT between risk strata are in the order of $200 \mu \mathrm{m}$, which is even below the theoretical, calculated axial resolution of typical ultrasound equipments used for measurement of CIMT. Thus, with the current techniques, measurement of CIMT is most useful when studying large groups of individuals, but it is unlikely that it will be incorporated into risk-assessment algorithms. Smaller studies have established that treatment effects of statins, niacin, beta-blockers, and calcium channel-blocking agents can be assessed by measuring $\mathrm{CIMT}^{13-18}$ but it has never been shown that these treatment effects translate into reductions in hard clinical endpoints, and thus CIMT has not been validated as a surrogate marker for use in cardiovascular intervention studies.

Intravascular ultrasound (IVUS) of the coronary tree has provided important new diagnostic information and insight into the pathogenesis of coronary artery disease. Other than angiography, greyscale IVUS allows for the assessment of plaque burden, thus taking into account not only plaques encroaching on the vascular lumen, but also plaques with eccentric remodelling. Atheroma burden measured with IVUS correlates closely with histology. ${ }^{19,20}$ In addition, characteristics of vulnerable plaques have been described to include eccentricity of the lesion, and echolucency in areas of positive remodelling. ${ }^{21}$ However, greyscale IVUS is limited for the exact analysis of plaque composition, with overlap in image characteristics between calcified and densely fibrotic tissue (strong signal reflection) on the one hand, and lipid-rich or fibrotic tissue and intraplaque haemorrhage (low signal reflection) on the other hand. Accordingly, non-vulnerable plaques in patients with stable angina have been found to share the aforementioned characteristics described in unstable lesions. ${ }^{22}$ The necessity for more precise evaluation of plaque composition has resulted in the development of methods that use the raw backscattered radiofrequency IVUS signal that is not subjected to the same limitations as greyscale data in terms of dynamic range, compression, and logarithmic transformation. Either the average power of the integrated backscatter $^{23}$ or spectral analysis of the frequency and power of backscatter (virtual histology) have been used to this end (Figure 1). ${ }^{24,25}$ For these techniques, mathematical algorithms for the classification of plaque components have been developed based on data aquired postmortem from human coronary arteries. Initial studies have subsequently shown good correlation to histology in ex vivo samples, ${ }^{23,25}$ and also in vivo when compared with histology of atherectomy samples. ${ }^{26}$ However, recent data from animal studies have questioned the accuracy of this technique for assessing plaque composition. ${ }^{27}$

\section{Arterial wall biomechanics}

Fibrosis, calcification, and smooth muscle cell proliferation cause changes in the thickness and biomechanical properties of arterial walls, which translate into increases in Young's elastic modulus and other parameters of deformability or stiffness. ${ }^{28}$ Changes in the elastic properties will also influence wave propagation in vessels, and the aortic wave front propagation velocity measured with either transoesophageal or transthoracic echocardiography has been used to determine aortic elastance. This parameter correlated with aortic atheroma burden and was independently predictive of cardiovascular events. ${ }^{29}$ With IVUS, the elastic properties of coronary artery walls have been assessed with elastography. This technique relies on cross-correlation analysis of radiofrequency signals to derive strain maps of the artery wall. ${ }^{30,31}$ Palpography is an identical technique that is used for interrogating only the luminal surface (to a depth of about $450 \mu \mathrm{m}$ ), and provides information on the surface of plaques, where rupture may occur. ${ }^{32,33}$ Strain values for potentially vulnerable plaques that contain necrotic cores, more inflammatory cells and less smooth muscle cells than stable lesions have consistently been shown to have higher strain values, ${ }^{31,32}$ and strain values have also been correlated to C-reactive protein levels. ${ }^{32}$ Recently, non-invasive elastography has also been used for the assessment of biomechanical properties of the carotid artery in small pilot studies. ${ }^{34,35}$

\section{Plaque neovascularization}

Atherosclerotic plaques rely on the formation of neovessels arising from adventitial vessels or from the lumen for growth beyond a volume at which nutritients supply from the arterial lumen or existing adventitial vessels is not sufficient anymore. It has been noted that the extent of neovascularization correlates not only with plaque volume, but also with features of instability like inflammatory state and morphological plaque rupture. ${ }^{36}$ Simple colour Doppler techniques have neither the sensitivity nor the spatial resolution to detect plaque 

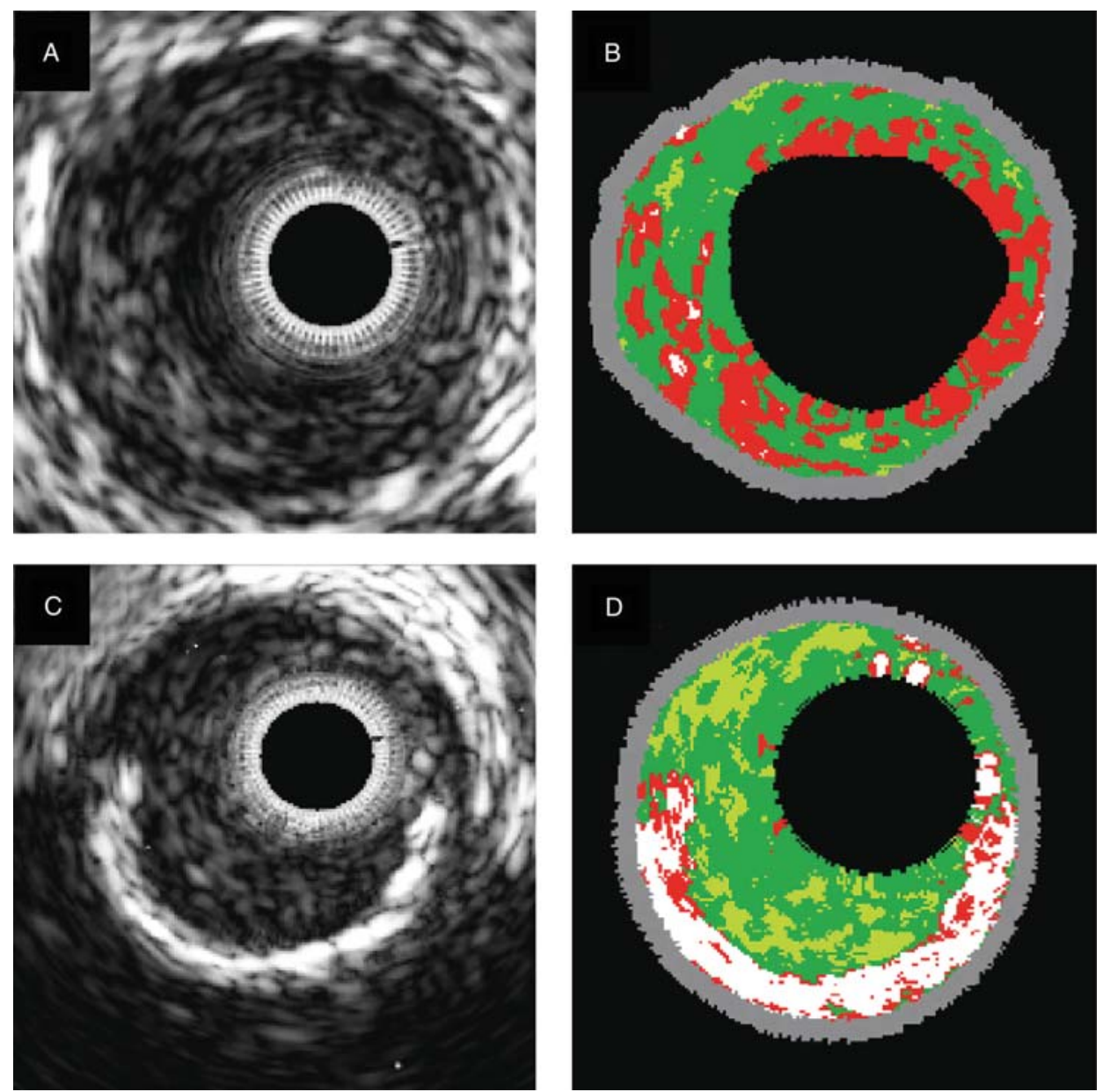

Figure 1 Examples of IVUS B-mode images and corresponding virtual histology (VH) images. On VH images, fibrous areas are marked in green, firbo-fatty in yellow, dense calcium in white, and necrotic core in red. (A) IVUS grayscale image of a thin-cap fibroatheroma. (B) Colour-coded, reconstructed VH image of (A) shows a large necrotic core whithout evidence of a fibrous cap. (C) IVUS grayscale image of a fibrocalcific atheroma. (D) Colour-coded VH image of (C) with the absence of a necrotic core. Note dense calcium at the bottom of the plaque, also visible in $(C)$. Reproduced from Nasu et al. ${ }^{68}$ with permission from BMJ Publishing Group Ltd.

neovascularization. Contrast-enhanced ultrasound has been used for the detection of plaque neovessels in the carotid arteries, ${ }^{37,38}$ and a semi-quantitative assessment of plaque neovascularization correlated to neovessels as visualized by CD31 staining on pathology specimens (Figure 2). ${ }^{38}$ Fundamental frequency IVUS imaging is not sensitive enough for detecting contrast agents in vasa vasorum in coronary arteries. However, recently harmonic imaging IVUS instrumentation has been developed and shown to be capable of detecting ultrasound contrast agent in the vasa vasorum of the atherosclerotic rabbit aorta. ${ }^{39}$

\section{Ultrasound molecular imaging of atherosclerosis}

Ultrasound molecular imaging uses microbubbles, other microparticles like echogenic liposomes, or acoustically active nanoparticles as tracers. These particles are targeted to specific molecular structures present on the cell surface in the tissue of interest. Targeting strategies for microbubbles exploit either the shell characteristics of microparticles for attachment to activated leukocytes within inflamed vessels ${ }^{40}$ or specific targeting by virtue of ligands that are conjugated to their surface. ${ }^{41}$ Targeting strategies for smaller micro- or nanoparticles rely on surface attachment of appropriate ligands. Antibodies, polysaccharides, and short peptide sequences have been used as targeting ligands. The concept of ultrasound molecular imaging is that, after intravenous injection, these microparticles bind to disease-specific epitopes and can then be imaged noninvasively in real time. When insonicated, microbubbles and, to a lesser extent, other accoustically active microparticles, emit soundwaves that can be detected by ultrasound systems. While there has been concern on signal damping caused by attachment to endothelial cells, in vitro studies have shown that antibody-mediated attachment to endothelial cells leads only to minimal dampening of their signal, but appears to diminish the rate of gas loss of these microbubbles. ${ }^{42}$ In the last few years, several studies have shown that with these tracers, ultrasound imaging of molecular targets that are relevant to the development of atherosclerosis is feasible.

\subsection{Molecular targets for ultrasound molecular imaging of atherosclerosis}

Microbubbles do not leave the intravascular space, ${ }^{43}$ and thus potential targets must by necessity be present on the 

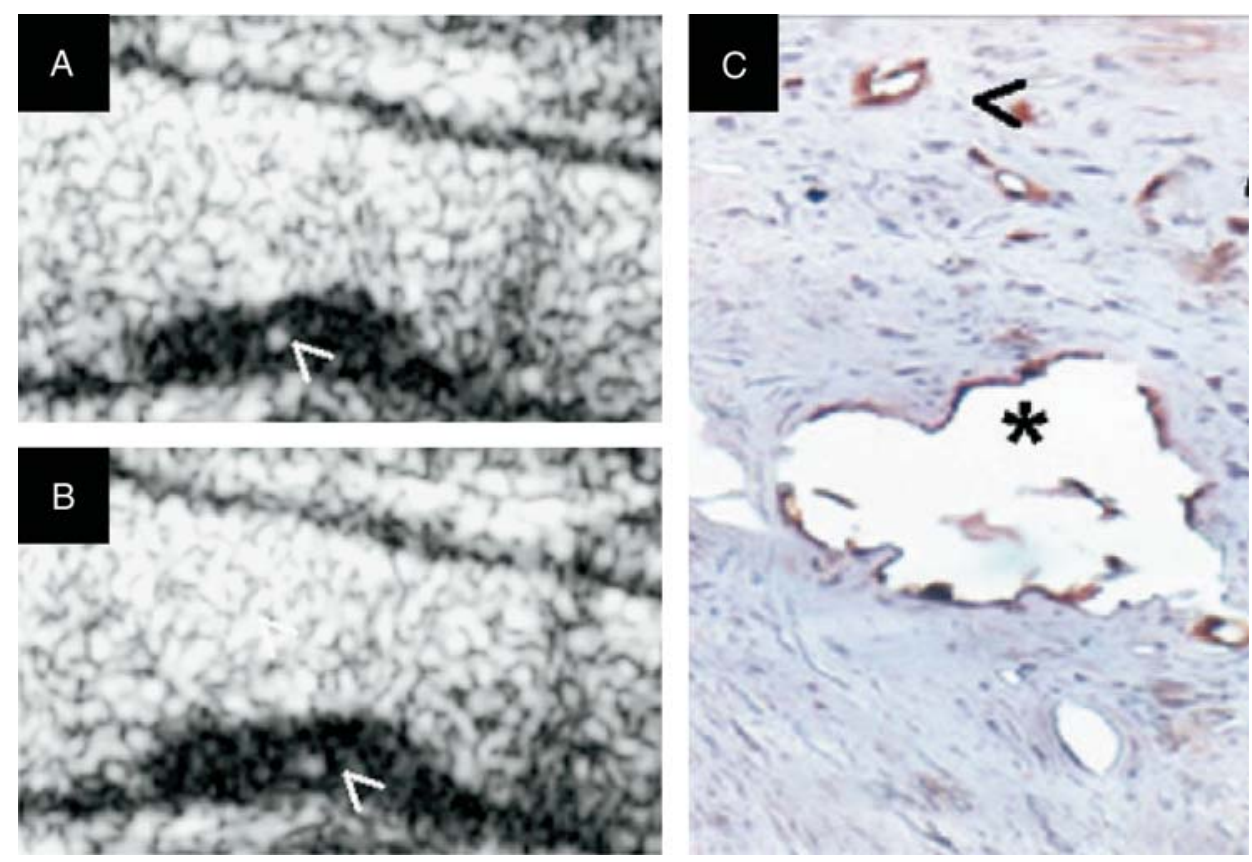

Figure 2 Plaque neovascularization detected by contrast-enhanced ultrasound. $(A)$ and $(B)$ are consecutive ultrasound frames of an atherosclerotic lesion in the carotid bulb showing microbubbles within the plaque (white arrowheads). (C) Immunohistological staining of the fibrous cap of the lesion imaged in ( $A$ ) and (B) showing positive staining for CD31 corresponding to a large first-order neovessel (asterisks) and a smaller second-order neovessel (arrowhead). Reproduced from Coli et al. ${ }^{38}$ with permission from Elsevier.

endothelial cell surface. In contrast, acoustically active microparticles or nanoemulsions can potentially leave the intravascular space and may allow acoustic targeting of extravascular structures. ${ }^{44,45}$

Inflammation plays an important role in the initiation and progression of cardiovascular disease and has been identified as a potential diagnostic target. Monocyte and lympohocyte recruitment to the vessel wall is one of the most important contributors to atherogenesis. These cells serve as a source for reactive oxygen species, pro-thrombotic compounds, pro-angiogenic growth factors and cytokines, vasoactive peptides, and a multitude of bioactive products that promote smooth muscle cell migration and further inflammatory cell recruitment. Monocyte and lymphocyte entry into the vessel wall is tightly regulated and mediated, in part, by the interaction between endothelial cell adhesion molecules expressed in inflamed regions and their counterligands on the leukocyte surface. P-selectin is a molecule that is responsible for initial capture and rapid rolling of monocytes and neutrophils on the microvascular endothelial surface by interacting with its glycosylated and fucosylated ligand PSGL-1. ${ }^{46}$ Cytokine-mediated P-selectin expression can be detected on circulating platelets and is associated with subclinical atherosclerotic disease in patients. ${ }^{47}$ Also, in mouse models of atherosclerosis, P-selectin-dependent monocyte rolling has been demonstrated on early atherosclerotic lesions. ${ }^{48}$ Vascular cell adhesion molecule 1 (VCAM-1), a cell adhesion molecule from the immunoglobuline gene superfamily promotes slow rolling and eventual firm attachment and diapedesis of neutrophils, monocytes, and lymphocytes by interacting with the heterodimeric integrin VLA-4 $(\alpha 4 \beta 1) .{ }^{46}$ In murine models of atherosclerotic disease, slow rolling of monocytes on the surface of atherosclerotic plaques appears to be largely mediated by VCAM-1. ${ }^{49}$ Intercellular cell adhesion molecule 1 (ICAM-1) is a second cell adhesion molecule from the immunoglobulin gene superfamily that has been implicated in monocyte recruitment in atherosclerosis. ${ }^{50}$ With regards to the use of these cell adhesion molecules as early markers of disease, VCAM- 1 has been demonstrated to be upregulated on the endothelium of Apo $\mathrm{E}-/$ - and LDL receptor (LDLR) - / - mice before the development of early atherosclerotic lesions, ${ }^{51,52}$ and homozygous VCAM-1 domain 4-deficient mice on a LDLR - / - background show a slower progression of atherosclerosis. ${ }^{53}$ Given the aforementioned role of VCAM-1 and P-selectin in the early pathogenesis of atherosclerosis, these markers are thought to be of potential value in the early assessment of the risk for the development of clinical atherosclerotic disease.

Other potential targets for the molecular assessment of atherosclerosis and/or atherosclerotic plaques include tissue factor (TF) and several molecular markers of angiogenesis. TF is not expressed on normal endothelial cells. However, upon stimulation with cytokines, biogenic amines such as serotonin or histamine, or by mediators such as oxidized LDL or vascular endothelial growth factor, expression of TF can be induced on endothelial cells. ${ }^{54}$ TF has been related to thrombotic complications after the disruption or erosion of atherosclerotic plaques. ${ }^{55}$ In addition, TF is involved in migratory and proliferative signalling to vascular smooth muscle cells and could potentially contribute to plaque progression and destabilization. Thus, there has been an interest in imaging the expression of TF in plaques for characterizing the plaque biology and risk for complications. Likewise, there is interest in imaging molecular markers of angiogenesis to detect pathological neovascularization associated with plaque progression and instability. Molecules expressed on the vascular endothelium that 

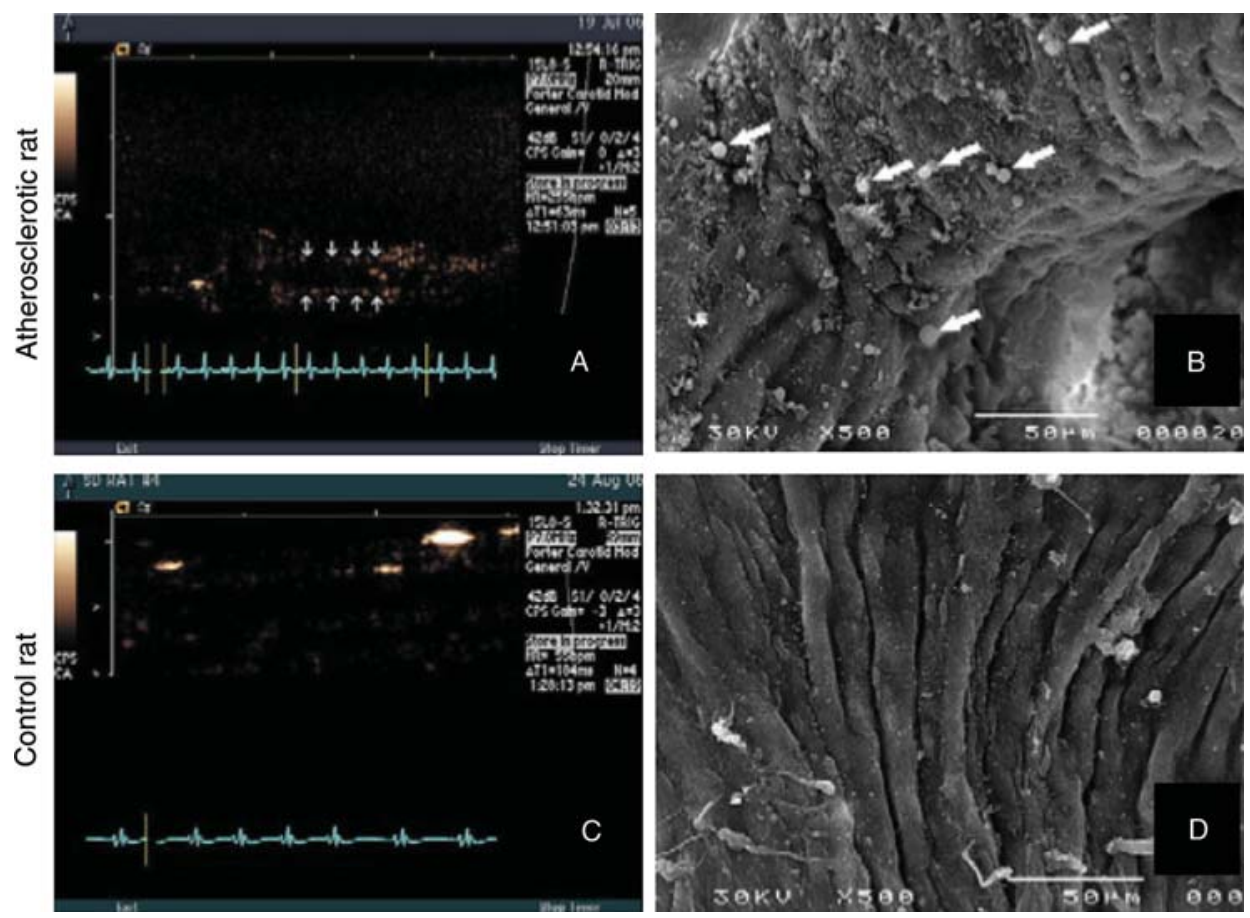

Figure 3 Ultrasound images at low mechanical index and scanning electron microscopy (SEM) of the aortic arch in an atherosclerotic rat ( $A, B)$ and a control rat $(C, D)$ after intravenous injection of PESDA microbubbles. These images illustrate signal from attached PESDA microbubbles in the atherosclerotic rat $(A)$ and microbubble retention viewed by SEM ( $B$; white arrows). In the control rat, no signal was detected on ultrasound $(C)$, and SEM confirmed the absence of attached microbubbles. Reproduced from Anderson et al. ${ }^{66}$ with permission.

could potentially be targeted for assessing plaque neovascularization include $\alpha_{v}$ integrins, $\alpha_{5}$ integrins, and VEGF receptors. Ultrasound probes for these targets ${ }^{56-58}$ have been developed and employed to detect tumour- or ischaemiamediated angiogenesis.

\subsection{Targeted microbubble attachment in high shear flow vessels}

The extent of retention of targeted microbubbles on a molecular marker is determined by ligand density on the tracer surface, by on- and off-rates of the ligands that are being used, the wall shear stress within the vasculature of interest, and potentially, by partitioning of the tracer in the bloodstream. For antibody-targeted microbubbles, the typical ligand density is $>50000$ antibodies per microbubble or a surface density of several 1000 antibodies per square micrometre. Ultrasound molecular imaging has been performed in a number of tissues (myocardium, skeletal muscle, kidney) relying on the selective retention of targeted microbubbles in the microvasculature, where wall shear stresses are low. ${ }^{41,57,59}$ Wall shear stress in the commonly used murine models of atherosclerosis is much higher, with peak systolic shear stress reaching up to 80 90 dynes $/ \mathrm{cm}^{2}, 60,61$ in the mouse aorta. Antibody-based microbubble targeting is the most common and most versatile technique for ultrasound molecular imaging. Given the low bond formation rate (low on-rate) of antibodies, there has been concern that this could potentially preclude bond formation in vessels with rapid transit rates and high shear stress. Indeed, flow chamber studies performed with variable shear stresses under continuous flow conditions have shown that the retention fraction of targeted microbubbles decreases dramatically at shear rates well below the peak shear rates described in the mouse aorta. ${ }^{62,63}$ However, arterial flow is pulsatile, and decreases to near zero during diastole in the murine aorta. Accordingly, flow chamber experiments with simulations of pulsatile flow have demonstrated microbubble attachment with only brief interruptions in high shear rate flow. ${ }^{63}$ Subsequent resumption of high shear rate flow does not detach these microbubbles, indicating excellent bond strength of attached antibodytargeted microbubbles. Thus, microbubble attachment does occur in high shear rate conditions provided that the flow is pulsatile. It is important to realize that wall shear rates are at least on the order of a magnitude lower in large arteries in humans, and therefore, with regards to potential clinical applications, attachment efficiency should be equivalent or better in humans when compared with small animal models of disease.

\subsection{Feasibility of ultrasound molecular imaging in atherosclerosis}

Microbubbles with a lipid or albumin shell have been shown to bind to activated monocytes and neutrophils via mechanisms that are mediated by complement $\mathrm{C} 3$ and/or attachment to Mac-1, and complement-mediated attachment to endothelial cells is possible for lipid-shelled microbubbles with a sufficiently negative surface charge. ${ }^{64}$ Attachment of perfluorocarbon-exposed sonicated dextrose albumin (PESDA) microbubbles to dysfunctional endothelium has also been shown to occur in a pig model of stretch-induced injury in the carotid arteries during hypertriglceridaemia. ${ }^{65}$ Subsequent experiments have shown that attachment of PESDA microbubbles to dysfunctional vascular endothelium is drastically reduced after complement depletion, and that extensive attachment also occurs in early atherosclerosis in the rat aorta (Figure 3). ${ }^{66}$ 

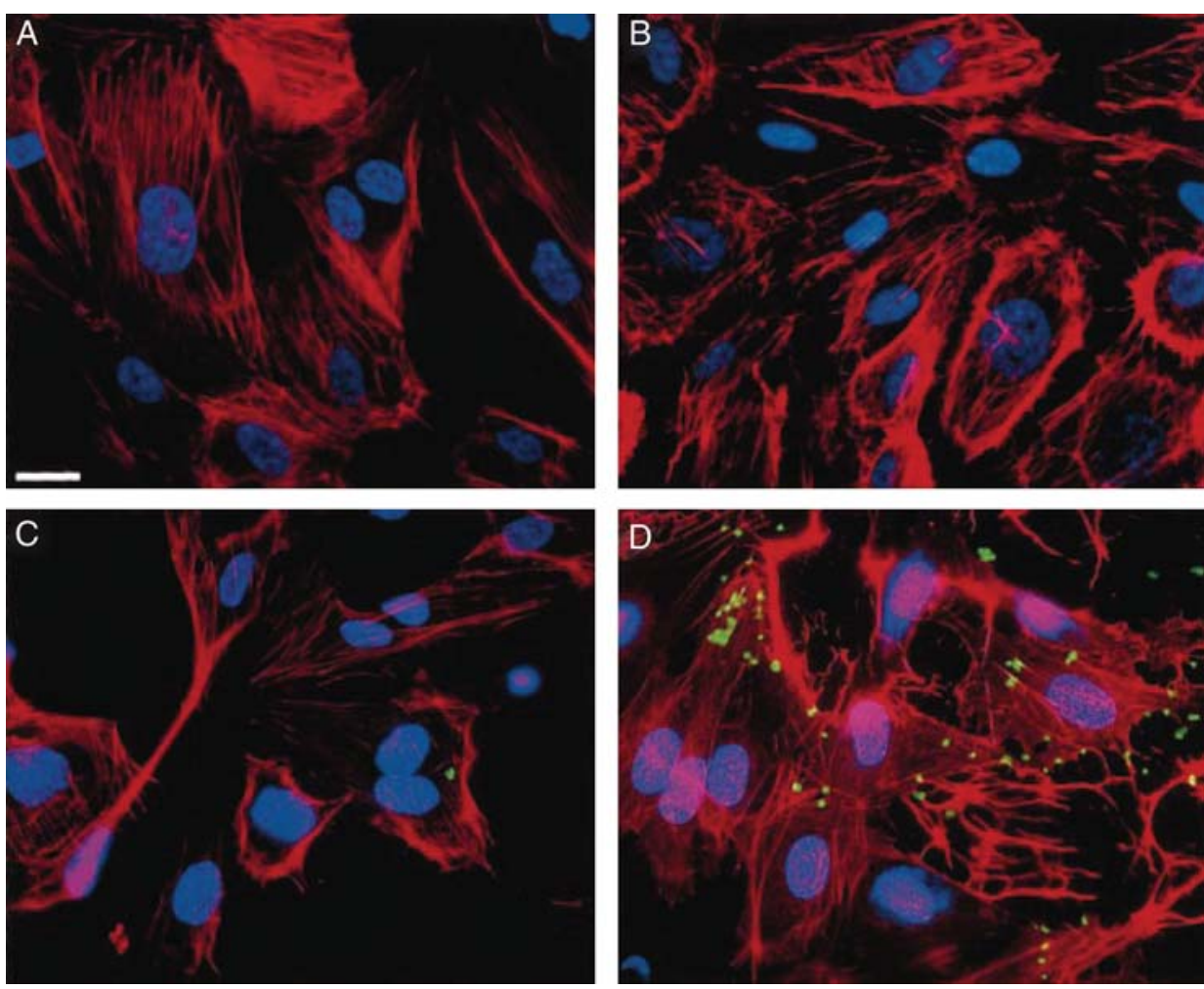

Figure 4 Fluorescence microscopy of endothelial cells after incubation with microbubbles bearing either a non-binding control antibody, or an antibody to ICAM-1. These images show absence of attachment of control microbubbles to non-activated $(A)$ and IL-1 $\beta$-activated endothelial cells $(B)$. Attachment of microbubbles targeted to ICAM-1 was low in non-activated endothelial cells $(C)$. After IL-1 $\beta$ activation, numerous targeted microbubbles were found attaching to endothelial cells $(D)$. Scale bar $=10 \mu \mathrm{m}$. Of note, the microbubbles have lost their gas and spherical shape owing to preparation for microscopy. Reproduced from Villanueva et al. ${ }^{67}$ with permission.

Specific binding of antibody-targeted microbubbles to endothelial cells has first been demonstrated for ICAM-1targeted microbubbles using in vitro experimental approaches (Figure 4). ${ }^{67}$ Early in vivo studies involved the intra-arterial injection of echogenic liposomes targeted to ICAM-1 in Yucatan miniswines, fed a hypercholesterolaemic diet after the induction of atherosclerotic plaques by endothelial denudement. Attachment of liposomes to plaques was then visualized using intravascular and transvascular ultrasound approaches. Later studies demonstrated selective attachment and imaging of the cell adhesion molecules ICAM- 1 and VCAM- 1 and also of TF after intra-arterial injections of targeted echogenic liposomes. ${ }^{44}$ Thus, in these studies several key mediators of atherosclerosis initiation and progression could be imaged, albeit with the drawback that very high intra-arterial doses of liposomes and invasive imaging methods were used.

Targeting of VCAM-1 in the aorta of atherosclerotic Apo E lipoprotein-deficient (Apo $\mathrm{E}-\mathrm{I}_{-}$) mice has also been recently accomplished with intravenously injected microbubbles. $^{63}$ ApoE $-/-$ mice develop atherosclerotic plaques in lesion-prone regions in the aortic arch, the degree of which can be modified by feeding the animals a normal chow or high cholesterol diet. Fluorescent microscopy showed selective attachment of VCAM-1targeted microbubbles in these models, and the extent of attachment correlated to the severity of plaque development. Similarly, non-invasive ultrasound imaging of the aortic arch showed a stepwise increase in VCAM-1-targeted microbubble signal according to disease phenotype. Thus, in these studies, disease severity could be non-invasively imaged by assessing the expression of VCAM-1 (Figure 5).

\subsection{Future directions in ultrasound molecular imaging of atherosclerosis}

While the aforementioned studies demonstrate the feasibility of ultrasound molecular imaging in the evaluation of atherosclerosis, no clinical studies using this technique have been performed thus far. For the initiation of clinical trials, several developments need to be made both for the contrast agents that are being used, as well as in the imaging technology for tracer detection. Most experimental studies have used simple biotion-streptavidin-biotin chemistry for ligand conjugation to the tracer surface. Streptavidin could potentially lead to binding of biotin in the body, which is needed for fatty acid synthesis and gluconeogenesis, and could thus have untoward effects. Alternative conjugation strategies that are expected to be safe for human use include amine or sulfhydryl covalent bonds. Likewise, there is concern about the use of antibodies in humans both in terms of safety and costs, and there is a need for the development of small-molecule, highly specific and inexpensive ligands which will most certainly include peptide molecules. For molecular imaging of atherosclerosis, attachment of the tracer to a relatively small area like the endothelial surface of a carotid artery, as opposed to the microvasculature in a tissue, is required, and only a limited amount of tracer particles will be available for detection. Thus, strategies for tracer detection above 

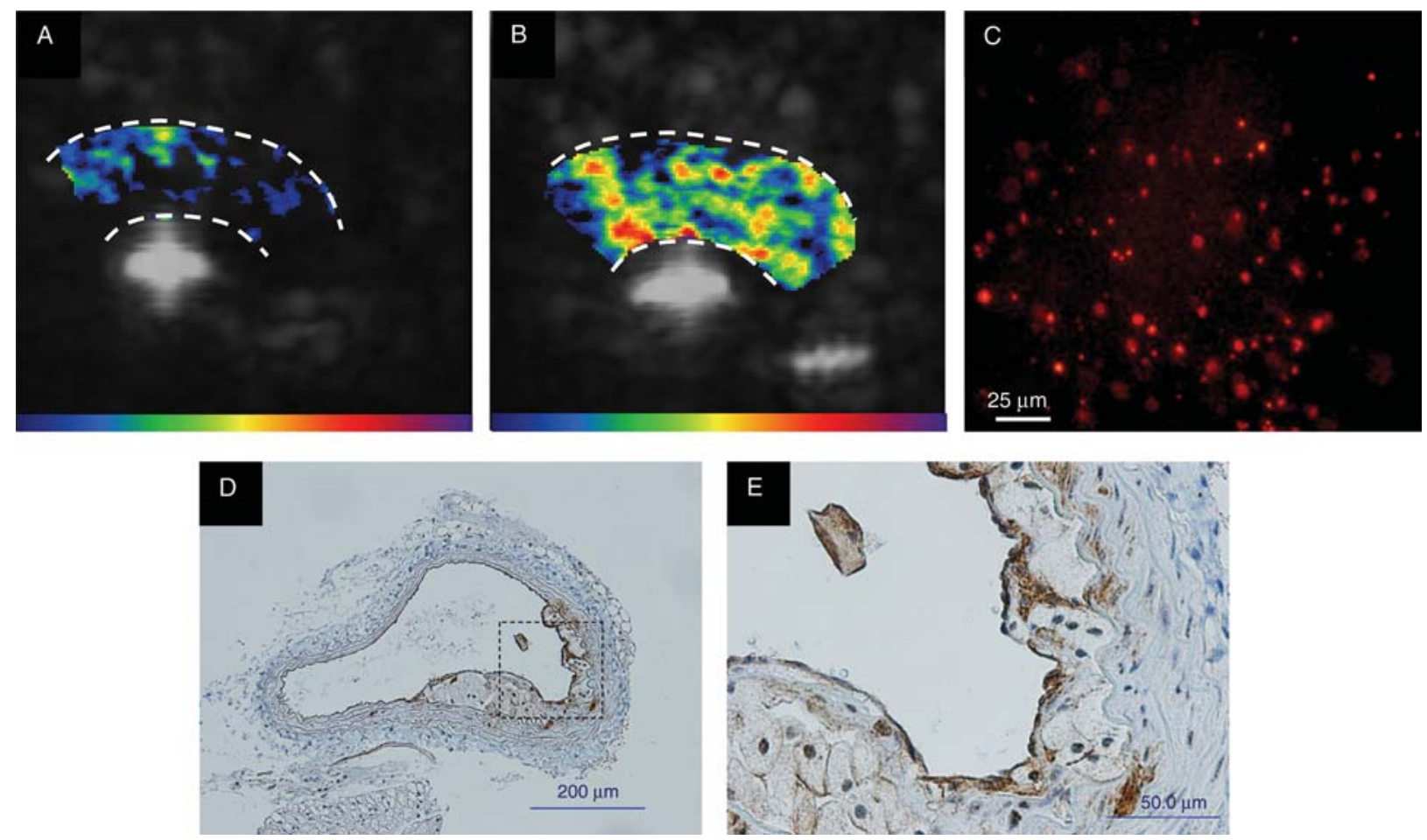

Figure 5 Examples of contrast-enhanced ultrasound targeted imaging of VCAM-1 in Apo E- / - mice on high cholesterol diet. These images show low signal from control microbubbles $(A)$ and high signal from VCAM-1-targeted microbubbles $(B)$. Fluorescent microscopy revealed extensive attachment of VCAM-1-targeted microbubbles to the aortic endothelium in Apo $\mathrm{E}-/$ - mice on high cholesterol diet $(C)$. Immunohistological staining for VCAM-1 in these mice revealed extensive plaque formation $(D)$ with expression of VCAM-1 both on the endothelial surface and on macrophages within the plaque $(E)$.

background noise in arteries with strong specular reflection need to be optimized. In addition, algorithms for online detection of attached microbubbles only are desirable. It should also be emphasized that once the aforementioned technological advances have been accomplished, initial clinical trials will most certainly include imaging of readily accessible vessels like the carotid arteries, and inflammatory or other molecular changes that are detected will serve as surrogate markers for cardiovascular risk. However, there is a keen interest in molecular imaging of the coronary arteries, especially for the detection of vulnerable plaques, that will make further refinement of ultrasound imaging equipment and tracer design necessary.

\section{Conclusions}

Enormous progress has been made in the last years in uncovering the molecular mechanisms that lead to initiation and progression of atherosclerosis. Undoubtedly, this research will eventually translate into new therapeutic approaches. This creates a need for the non-invasive assessment of atherosclerosis on a subcellular level for earlier recognition of disease or for better evaluation of risk and disease progression in established disease, as well as for the assessment of the effect of existing and new, costly therapies. Atherosclerosis and risk factors for the development of atherosclerosis are highly prevalent, and therefore such non-invasive imaging techniques need to offer high throughput, widely available and inexpensive screening capabilities. The ultrasound imaging techniques described in this review are well positioned for eventually satisfying these requirements.

\section{Funding}

B.A.K. is supported by grant 32323B_123819/1 from the Swiss National Science Foundation and by a grant from the Swiss Heart Foundation.

Conflict of interest: none declared.

\section{References}

1. Ford ES, Capewell S. Coronary heart disease mortality among young adults in the U.S. from 1980 through 2002: concealed leveling of mortality rates. J Am Coll Cardiol 2007;50:2128-2132.

2. Lloyd-Jones D, Adams R, Carnethon M, De Simone G, Ferguson TB, Flegal $\mathrm{K}$ et al. Heart Disease and Stroke Statistics-2009 Update. A Report From the American Heart Association Statistics Committee and Stroke Statistics Subcommittee. Circulation 2009;119:480-486.

3. Flegal KM, Carroll MD, Ogden CL, Johnson CL. Prevalence and trends in obesity among US adults, 1999-2000. JAMA 2002;288:1723-1727.

4. Ford ES, Giles WH, Mokdad AH. Increasing prevalence of the metabolic syndrome among US Adults. Diabetes Care 2004;27:2444-2449.

5. Jacobson TA, Griffiths GG, Varas C, Gause D, Sung JC, Ballantyne CM. Impact of evidence-based 'clinical judgment' on the number of American adults requiring lipid-lowering therapy based on updated NHANES III data. National Health and Nutrition Examination Survey. Arch Intern Med 2000; 160:1361-1369.

6. Stork S, van den Beld AW, von Schacky C, Angermann CE, Lamberts SW, Grobbee DE et al. Carotid artery plaque burden, stiffness, and mortality risk in elderly men: a prospective, population-based cohort study. Circulation 2004; 110:344-348.

7. Chan SY, Mancini GB, Kuramoto L, Schulzer M, Frohlich J, Ignaszewski A. The prognostic importance of endothelial dysfunction and carotid atheroma burden in patients with coronary artery disease. J Am Coll Cardiol 2003;42:1037-1043.

8. Chambless LE, Folsom AR, Davis V, Sharrett R, Heiss G, Sorlie P et al. Risk factors for progression of common carotid atherosclerosis: the Atherosclerosis Risk in Communities Study, 1987-1998. Am J Epidemiol 2002; 155:38-47. 
9. Chambless LE, Heiss G, Folsom AR, Rosamond W, Szklo M, Sharrett AR et al. Association of coronary heart disease incidence with carotid arterial wall thickness and major risk factors: the Atherosclerosis Risk in Communities [ARIC] Study, 1987-1993. Am J Epidemiol 1997;146: 483-494.

10. Chambless LE, Folsom AR, Clegg LX, Sharrett AR, Shahar E, Nieto FJ et al. Carotid wall thickness is predictive of incident clinical stroke: the Atherosclerosis Risk in Communities [ARIC] study. Am J Epidemiol 2000;151: 478-487.

11. O'Leary DH, Polak JF, Kronmal RA, Manolio TA, Burke GL, Wolfson SK. Carotid-artery intima and media thickness as a risk factor for myocardial infarction and stroke in older adults. Cardiovascular Health Study Collaborative Research Group. N Engl J Med 1999;340:14-22.

12. Bots ML, Hoes AW, Koudstaal PJ, Hofman A, Grobbee DE. Common carotid intima-media thickness and risk of stroke and myocardial infarction: the Rotterdam Study. Circulation 1997;96:1432-1437.

13. Taylor AJ, Kent SM, Flaherty PJ, Coyle LC, Markwood TT, Vernalis MN. ARBITER: Arterial Biology for the Investigation of the Treatment Effects of Reducing Cholesterol: a randomized trial comparing the effects of atorvastatin and pravastatin on carotid intima medial thickness. Circulation 2002;106:2055-2060.

14. Taylor AJ, Sullenberger LE, Lee HJ, Lee JK, Grace KA. Arterial Biology for the Investigation of the Treatment Effects of Reducing Cholesterol [ARBITER] 2: a double-blind, placebo-controlled study of extendedrelease niacin on atherosclerosis progression in secondary prevention patients treated with statins. Circulation 2004;110:3512-3517.

15. Hedblad B, Wikstrand J, Janzon L, Wedel H, Berglund G. Low-dose metoprolol $\mathrm{CR} / \mathrm{XL}$ and fluvastatin slow progression of carotid intima-media thickness: main results from the Beta-Blocker Cholesterol-Lowering Asymptomatic Plaque Study (BCAPS). Circulation 2001;103: $1721-1726$

16. Wiklund O, Hulthe J, Wikstrand J, Schmidt C, Olofsson SO, Bondjers G. Effect of controlled release/extended release metoprolol on carotid intima-media thickness in patients with hypercholesterolemia: a 3-year randomized study. Stroke 2002;33:572-577.

17. Pitt $B$, Byington RP, Furberg CD, Hunninghake DB, Mancini GB, Miller ME et al. Effect of amlodipine on the progression of atherosclerosis and the occurrence of clinical events. PREVENT Investigators. Circulation 2000; 102:1503-1510.

18. Smilde TJ, van WS, Wollersheim H, Trip MD, Kastelein JJ, Stalenhoef AF. Effect of aggressive versus conventional lipid lowering on atherosclerosis progression in familial hypercholesterolaemia (ASAP): a prospective, randomised, double-blind trial. Lancet 2001;357:577-581.

19. Potkin BN, Bartorelli AL, Gessert JM, Neville RF, Almagor Y, Roberts WC et al. Coronary artery imaging with intravascular high-frequency ultrasound. Circulation 1990;81:1575-1585.

20. Nishimura RA, Edwards WD, Warnes CA, Reeder GS, Holmes DR, Tajik AJ et al. Intravascular ultrasound imaging: in vitro validation and pathologic correlation. J Am Coll Cardiol 1990;16:145-154.

21. Yamagishi M, Terashima M, Awano K, Kijima M, Nakatani S, Daikoku S et al. Morphology of vulnerable coronary plaque: insights from follow-up of patients examined by intravascular ultrasound before an acute coronary syndrome. J Am Coll Cardiol 2000;35:106-111.

22. Schoenhagen P, Stone GW, Nissen SE, Grines CL, Griffin J, Clemson BS et al. Coronary plaque morphology and frequency of ulceration distant from culprit lesions in patients with unstable and stable presentation. Arterioscler Thromb Vasc Biol 2003;23:1895-1900.

23. Kawasaki M, Takatsu H, Noda T, Sano K, Ito Y, Hayakawa K et al. In vivo quantitative tissue characterization of human coronary arterial plaques by use of integrated backscatter intravascular ultrasound and comparison with angioscopic findings. Circulation 2002;105:2487-2492.

24. Nair A, Kuban BD, Obuchowski N, Vince DG. Assessing spectral algorithms to predict atherosclerotic plaque composition with normalized and raw intravascular ultrasound data. Ultrasound Med Biol 2001;27:1319-1331.

25. Nair A, Kuban BD, Tuzcu EM, Schoenhagen P, Nissen SE, Vince DG. Coronary plaque classification with intravascular ultrasound radiofrequency data analysis. Circulation 2002;106:2200-2206.

26. Nasu K, Tsuchikane E, Katoh O, Vince DG, Virmani R, Surmely JF et al. Accuracy of in vivo coronary plaque morphology assessment: a validation study of in vivo virtual histology compared with in vitro histopathology. J Am Coll Cardiol 2006;47:2405-2412.

27. Granada JF, Wallace-Bradley D, Win HK, Alviar CL, Builes A, Lev El et al. In vivo plaque characterization using intravascular ultrasound-virtual histology in a porcine model of complex coronary lesions. Arterioscler Thromb Vasc Biol 2007;27:387-393.
28. Lee RT, Grodzinsky AJ, Frank EH, Kamm RD, Schoen FJ. Structuredependent dynamic mechanical behavior of fibrous caps from human atherosclerotic plaques. Circulation 1991;83:1764-1770.

29. Hunziker PR, Imsand C, Keller D, Hess N, Barbosa V, Nietlisbach F et al. Bedside quantification of atherosclerosis severity for cardiovascular risk stratification: a prospective cohort study. J Am Coll Cardiol 2002;39: 702-709.

30. de Korte CL, van der Steen AF, Cespedes El, Pasterkamp G. Intravascular ultrasound elastography in human arteries: initial experience in vitro. Ultrasound Med Biol 1998;24:401-408.

31. Schaar JA, de Korte CL, Mastik F, Strijder C, Pasterkamp G, Boersma E et al. Characterizing vulnerable plaque features with intravascular elastography. Circulation 2003;108:2636-2641.

32. Schaar JA, Regar E, Mastik F, McFadden EP, Saia F, Disco C et al. Incidence of high-strain patterns in human coronary arteries: assessment with three-dimensional intravascular palpography and correlation with clinical presentation. Circulation 2004;109:2716-2719.

33. Schaar JA, van der Steen AF, Mastik F, Baldewsing RA, Serruys PW. Intravascular palpography for vulnerable plaque assessment. J Am Coll Cardiol 2006;47:C86-C91.

34. Maurice RL, Soulez G, Giroux MF, Cloutier G. Noninvasive vascular elastography for carotid artery characterization on subjects without previous history of atherosclerosis. Med Phys 2008;35:3436-3443.

35. Schmitt C, Soulez G, Maurice RL, Giroux MF, Cloutier G. Noninvasive vascular elastography: toward a complementary characterization tool of atherosclerosis in carotid arteries. Ultrasound Med Biol 2007;33: 1841-1858.

36. Moreno PR, Purushothaman KR, Fuster V, Echeverri D, Truszczynska H, Sharma SK et al. Plaque neovascularization is increased in ruptured atherosclerotic lesions of human aorta: implications for plaque vulnerability. Circulation 2004;110:2032-2038.

37. Vicenzini E, Giannoni MF, Puccinelli F, Ricciardi MC, Altieri M, DiPiero V et al. Detection of carotid adventitial vasa vasorum and plaque vascularization with ultrasound cadence contrast pulse sequencing technique and echo-contrast agent. Stroke 2007;38:2841-2843.

38. Coli S, Magnoni M, Sangiorgi G, Morrocco-Trischitta MM, Melisurgo G, Mauriello A et al. Contrast-enhanced ultrasound imaging of intraplaque neovascularization in carotid arteries: correlation with histology and plaque echogenicity. J Am Coll Cardiol 2008;52:223-230.

39. Goertz DE, Frijlink ME, Tempel D, van Damme LC, Krams R, Schaar JA et al. Contrast harmonic intravascular ultrasound: a feasibility study for vasa vasorum imaging. Invest Radiol 2006;41:631-638.

40. Christiansen JP, Leong-Poi H, Klibanov AL, Kaul S, Lindner JR. Noninvasive imaging of myocardial reperfusion injury using leukocyte-targeted contrast echocardiography. Circulation 2002;105:1764-1767.

41. Lindner JR, Song J, Christiansen J, Klibanov AL, Xu F, Ley K. Ultrasound assessment of inflammation and renal tissue injury with microbubbles targeted to P-selectin. Circulation 2001;104:2107-2112.

42. Lankford M, Behm CZ, Yeh J, Klibanov AL, Robinson P, Lindner JR. Effect of microbubble ligation to cells on ultrasound signal enhancement: implications for targeted imaging. Invest Radiol 2006;41:721-728.

43. Jayaweera AR, Edwards N, Glasheen WP, Villanueva FS, Abbott RD, Kaul S. In vivo myocardial kinetics of air-filled albumin microbubbles during myocardial contrast echocardiography. Comparison with radiolabeled red blood cells. Circ Res 1994;74:1157-1165.

44. Hamilton AJ, Huang SL, Warnick D, Rabbat M, Kane B, Nagaraj A et al. Intravascular ultrasound molecular imaging of atheroma components in vivo. J Am Coll Cardiol 2004;43:453-460.

45. Lanza GM, Abendschein DR, Hall CS, Marsh JN, Scott MJ, Scherrer DE et al. Molecular imaging of stretch-induced tissue factor expression in carotid arteries with intravascular ultrasound. Invest Radiol 2000;35: 227-234.

46. Yang J, Hirata T, Croce K, Merrill-Skoloff G, Tchernychev B, Williams E et al. Targeted gene disruption demonstrates that $\mathrm{P}$-selectin glycoprotein ligand 1 [PSGL-1] is required for P-selectin-mediated but not E-selectin-mediated neutrophil rolling and migration. J Exp Med 1999; 190:1769-1782.

47. Shimizu M, Kohara S, Yamamoto M, Ando Y, Haida M, Shinohara Y. Significant relationship between platelet activation and intra-media thickness of the carotid artery in patients with ischemic cerebrovascular disease. Thromb Res 2006;117:647-652.

48. Ramos CL, Huo Y, Jung U, Ghosh S, Manka DR, Sarembock IJ et al. Direct demonstration of P-Selectin- and VCAM-1-dependent mononuclear cell rolling in early atherosclerotic lesions of apolipoprotein e-deficient mice. Circ Res 1999;84:1237-1244. 
49. Dansky HM, Barlow CB, Lominska C, Sikes JL, Kao C, Weinsaft J et al. Adhesion of monocytes to arterial endothelium and initiation of atherosclerosis are critically dependent on vascular cell adhesion molecule-1 gene dosage. Arterioscler Thromb Vasc Biol 2001;21:1662-1667.

50. Walpola PL, Gotlieb Al, Cybulsky MI, Langille BL. Expression of ICAM-1 and VCAM-1 and monocyte adherence in arteries exposed to altered shear stress. Arterioscler Thromb Vasc Biol 1995;15:2-10.

51. liyama K, Hajra L, liyama M, Li H, DiChiara M, Medoff BD et al. Patterns of vascular cell adhesion molecule-1 and intercellular adhesion molecule-1 expression in rabbit and mouse atherosclerotic lesions and at sites predisposed to lesion formation. Circ Res 1999;85:199-207.

52. Nakashima Y, Raines EW, Plump AS, Breslow JL, Ross R. Upregulation of VCAM-1 and ICAM-1 at atherosclerosis-prone sites on the endothelium in the ApoE-deficient mouse. Arterioscler Thromb Vasc Biol 1998;18: 842-851.

53. Cybulsky MI, liyama K, Li H, Zhu S, Chen M, liyama M et al. A major role for VCAM-1, but not ICAM-1, in early atherosclerosis. J Clin Invest 2001; 107:1255-1262.

54. Steffel J, Luscher TF, Tanner FC. Tissue factor in cardiovascular diseases: molecular mechanisms and clinical implications. Circulation 2006;113: 722-731.

55. Marmur JD, Thiruvikraman SV, Fyfe BS, Guha A, Sharma SK, Ambrose JA et al. Identification of active tissue factor in human coronary atheroma. Circulation 1996;94:1226-1232.

56. Leong-Poi H, Christiansen J, Heppner P, Lewis CW, Klibanov AL, Kaul S et al. Assessment of endogenous and therapeutic arteriogenesis by contrast ultrasound molecular imaging of integrin expression. Circulation 2005; 111:3248-3254.

57. Behm CZ, Kaufmann BA, Carr C, Lankford M, Sanders JM, Rose CE et al. Molecular imaging of endothelial vascular cell adhesion molecule-1 expression and inflammatory cell recruitment during vasculogenesis and ischemia-mediated arteriogenesis. Circulation 2008;117: 2902-2911.

58. Willmann JK, Paulmurugan R, Chen K, Gheysens O, Rodriguez-Porcel M, Lutz AM et al. US imaging of tumor angiogenesis with microbubbles targeted to vascular endothelial growth factor receptor type 2 in mice. Radiology 2008;246:508-518.
59. Kaufmann BA, Lewis C, Xie A, Mirza-Mohd A, Lindner JR. Detection of recent myocardial ischaemia by molecular imaging of $\mathrm{P}$-selectin with targeted contrast echocardiography. Eur Heart J 2007;28:2011-2017.

60. Eriksson EE, Werr J, Guo Y, Thoren P, Lindbom L. Direct observations in vivo on the role of endothelial selectins and alpha[4] integrin in cytokine-induced leukocyte-endothelium interactions in the mouse aorta. Circ Res 2000;86:526-533.

61. Greve JM, Les AS, Tang BT, Draney Blomme MT, Wilson NM, Dalman RL et al. Allometric scaling of wall shear stress from mice to humans: quantification using cine phase-contrast MRI and computational fluid dynamics. Am J Physiol Heart Circ Physiol 2006;291:H1700-H1708.

62. Takalkar AM, Klibanov AL, Rychak JJ, Lindner JR, Ley K. Binding and detachment dynamics of microbubbles targeted to P-selectin under controlled shear flow. J Control Release 2004;96:473-482.

63. Kaufmann BA, Sanders JM, Davis C, Xie A, Aldred P, Sarembock IJ et al. Molecular imaging of inflammation in atherosclerosis with targeted ultrasound detection of vascular cell adhesion molecule-1. Circulation 2007; 116:276-284.

64. Lindner JR, Coggins MP, Kaul S, Klibanov AL, Brandenburger GH, Ley K. Microbubble persistence in the microcirculation during ischemia/ reperfusion and inflammation is caused by integrin- and complementmediated adherence to activated leukocytes. Circulation 2000;101: 668-675.

65. Tsutsui JM, Xie F, Cano M, Chomas J, Philipps P, Radio SJ et al. Detection of retained microbubbles in carotid arteries with real-time low mechanical index imaging in the setting of endothelial dysfunction. $J$ Am Coll Cardiol 2004;44:1036-1046.

66. Anderson DR, Tsutsui JM, Xie F, Radio SJ, Porter TR. The role of complement in the adherence of microbubbles to dysfunctional arterial endothelium and atherosclerotic plaque. Cardiovasc Res 2007;73: 597-606.

67. Villanueva FS, Jankowski RJ, Klibanov S, Pina ML, Alber SM, Watkins SC et al. Microbubbles targeted to intercellular adhesion molecule-1 bind to activated coronary artery endothelial cells. Circulation 1998;98:1-5.

68. Nasu K, Tsuchikane E, Katoh O, Fujita H, Surmely JF, Ehara M et al. Plaque characterisation by virtual histology intravascular ultrasound analysis in patients with type 2 diabetes. Heart 2008;94:429-433. 